\title{
Influence de la nature des granulats sur le comportement rhéologique du béton à hautes températures
}

\author{
Zine El abidine Rahmouni ${ }^{1}$, Nadia Tebbal ${ }^{2}$ et Hassan Haroun abdellah ${ }^{3}$ \\ ${ }^{1,3}$ Université de M'sila, Département de Génie Civil, Algérie \\ ${ }^{2}$ Université de M'sila, Institut de gestion des techniques urbaines, Algérie.
}

\begin{abstract}
Résumé. Le béton est largement utilisé comme matériau de structure dans les ouvrages de génie civil tels que les tunnels. La prise en compte du comportement du béton soumis à des températures élevées est apparue dernièrement comme un problème majeur. Cette problématique concerne aussi bien des situations de service dans le cas des structures de stockage de déchets radioactifs que des situations d'accident, mises en exergue par les cas récents d'incendie dans des tunnels. Ce travail de recherche basé sur une approche expérimentale, analyse l'influence de la nature des granulats sur le comportement thermo-mécanique du béton soumis à une température élevée. Des bétons formulés avec deux granulats (calcaire concassé et silico-calcaire) subissent des cycles de chauffage/refroidissement à une vitesse de $10^{\circ} \mathrm{C} / \mathrm{min}$ à $200{ }^{\circ} \mathrm{C}, 400{ }^{\circ} \mathrm{C}$ et $900{ }^{\circ} \mathrm{C}$. Nous étudions l'évolution de la fissuration, des propriétés thermiques, physiques et mécaniques des bétons en fonction des températures appliquées. Pour la nature de granulat siliceux, le silex présente une instabilité thermique se traduisant par un éclatement à partir de $300^{\circ} \mathrm{C}$ alors que le granulat calcaire présente aussi une instabilité à cause des phénomènes de décarbonatation/hydratation mais seulement pour le cycle de chauffage/refroidissement à $750^{\circ} \mathrm{C}$.
\end{abstract}

Mots clés : Béton, Température élevée, Écaillage, Granulats, Sable siliceux, Sable concassé.

\section{Introduction}

Le béton est un matériau composite, constitué principalement de pâte de ciment et de granulats, qui est abondamment utilisé dans la construction de bâtiment et d'ouvrages en génie civil. Ce matériau peut être soumis à des températures élevées dans le cas extrêmes d'incendies. En cas de tels événement, le béton peut présenté une instabilité thermique à partir d'une certaine température. Il en résulte d'importantes dégradations du béton qui compromettent la sécurité de l'utilisation des ouvrages (les tunnels sous la Manche (1996et 2008), du Mont Blanc (1999) et du Fréjus (2005) en France, du Tauern (1999) en Autriche. les bétons après ces incendies montrent de forts endommagements de leur structure. on Observe l'apparition d'écaillage, d'éclatement ou de fissures.

De nombreux travaux de recherches ont été entrepris pour comprendre les processus physiques à l'origine des dommages dans le béton. On peut citer le rôle d'un processus thermomécanique où l'éjection de matériaux serait générée par des contraintes de compression élevée aux surfaces exposé au feu. De plus il y a un processus thermo-hydrique qui induit des surpressions de vapeur d'eau comparables à la résistance en traction du matériau. Il existe aussi une instabilité thermique liée aux granulats. La plupart des études réalisées sur l'endommagement se sont thermique du béton se sont intéressées à la pâte de ciment. En effet c'est au sein de cette pâte de ciment que se déroulent les principaux phénomènes de déshydratation et d'expulsion de l'humidité à l'origine des dégradations des bétons. Depuis une quinzaine d'années, les travaux de recherche ont permis d'améliorer la compréhension de l'évolution des propriétés physico chimiques et microstructurales de la phase liante. Moins d'étude se sont intéressées à l'influence de la nature des granulats sur le comportement des bétons à température élevée. Certains travaux montrent que le béton de granulats siliceux a une plus faible résistance aux sollicitations thermiques que le béton de granulats 
calcaire. La diminution de résistance en compression du béton de granulat calcaire est observée à une température plus élevée que celle du béton de granulats de granulats siliceux. Cette observation est généralement attribuée à la plus grande expansion thermique de granulats siliceux et à la l'augmentation de volume associée à la transition de phase de quartz $\alpha$ en quartz $\beta$ à $573^{\circ} \mathrm{C}$.

Certains auteurs expliquent aussi que la chaleur spécifique du granulat calcaire est approximativement dix fois celle nécessaire pour produire la même augmentation de température dans le granulat siliceux au dessus de $600^{\circ} \mathrm{C}$. Le granulat calcaire peut augmenter l'endurance $\mathrm{du}$ béton au feu par rapport au granulat siliceux .Par contre d'autre auteurs ont montré que le béton de granulat siliceux peut avoir un meilleur comportement résiduel mécanique que celui du béton de granulat calcaire [Zhi 2011].

Let travail présenté dans ce manuscrit a donc pour objet d'améliorer la compréhension de l'influence de la nature minéralogique des granulats sur le comportement de béton à haute température.

\section{Matériaux utilisés}

\subsection{Ciment}

IL provident de la cimenterie de Lafarge de M'sila. Ce ciment de référence CEM I 42.5. Il a une surface spécifique Blaine de $3585 \mathrm{~cm}^{2} / \mathrm{g}$ et une densité de 3,2 . La composition chimique et celle minéralogique selon les formules de Bogue sont données par le tableau 1.

Tableau 1. Composition chimiques et minéralogique du ciment

\begin{tabular}{|c|c|c|}
\hline \multirow{4}{*}{$\begin{array}{c}\text { Composition } \\
\text { chimiques }\end{array}$} & \multicolumn{2}{|c|}{ Compositions (\%) } \\
\cline { 2 - 3 } & $\mathrm{SiO}_{2}$ & 22.07 \\
\cline { 2 - 3 } & $\mathrm{CaO}$ & 63.83 \\
\cline { 2 - 3 } & $\mathrm{Al}_{2} \mathrm{O}_{3}$ & 6.41 \\
\cline { 2 - 3 } & $\mathrm{FeO}_{3}$ & 4.16 \\
\cline { 2 - 3 } & $\mathrm{MgO}$ & 0.21 \\
\cline { 2 - 3 } & $\mathrm{SO} 3$ & 1.49 \\
\cline { 2 - 3 } & $\mathrm{MnO}$ & $/ / / / /$ \\
\hline \multirow{3}{*}{$\begin{array}{c}\text { Compositions } \\
\text { minéralogiques }\end{array}$} & $\mathrm{C} 3 \mathrm{~S}$ & 60.3 \\
\cline { 2 - 3 } & $\mathrm{C} 2 \mathrm{~S}$ & 17.4 \\
\cline { 2 - 3 } & $\mathrm{C} 3 \mathrm{~A}$ & 6.13 \\
\cline { 2 - 3 } & $\mathrm{C} 4 \mathrm{AF}$ & 11.97 \\
\hline
\end{tabular}

\subsection{L'eau}

L'eau utilise est celle du réseau de distribution en eau potable de la Wilaya de M'sila. sa masse volumique utilisée dans les formulations est de $1000 \mathrm{~kg} / \mathrm{m}^{3}$.

\subsection{Le superplastifiant}

L'adjuvant utilise dans la confection de nos béton pour assure une fluidité satisfaisante de mise en oeuvre est le superplastifiant "Medaplast SP40" appartenant à la famille des superplastifiants / hauts réducteurs d'eau. Sa densité égale à 1.22 .

\subsection{Les granulats}

Le choix des sables utilises pour la confection des trois catégories de béton est guide par l'objectif de cette étude visant à estimer l'influence de la nature minéralogique des granulats sur le comportement des bétons à température élevée. Nous avons donc choisi trois types de sables de natures minéralogiques différentes qui correspondent aux trois classes généralement admises dans le domaine de génie civil. Ce sont respectivement des sables concassés provenant du calcaire massif (COSIDER, BBA), des granulats alluvionnaires semi concassés silico- calcaire (COSIDER de BBA) + (rivière de BOUSAADA) et des granulats alluvionnaires siliceux roulé (rivière de BOUSAADA). Leurs classes granulaires sont de $0 / 5 \mathrm{~mm}$.

Tableau 2. Paramètres physiques des sables.

\begin{tabular}{|c|c|c|c|}
\hline Type de sable & SD & SC & SDC \\
\hline $\begin{array}{c}\text { Masse volumique } \\
\text { apparente (kg/m3) }\end{array}$ & 1500 & 1550 & 1820 \\
\hline $\begin{array}{c}\text { Masse volumique absolus } \\
\text { (kg/m3) }\end{array}$ & 2500 & 2700 & 2630 \\
\hline Module de finesse & 1.87 & 2.1 & 1.8 \\
\hline Compacité (\%) & 66 & 65 & 69.21 \\
\hline Porosité (\%) & 34 & 35 & 30.79 \\
\hline $\begin{array}{c}\text { Equivalent de sable } \\
(\%)\end{array}$ & 75 & 98.96 & $1 / / / /$ \\
\hline
\end{tabular}

Les granulats utilisés sont des granulats concassés $(3 / 8$ et $8 / 15)$. Ils viennent de carrière COSIDER. Leurs densités absolue respectivement $\rho s=2,50$ et $\rho g=2,52$.

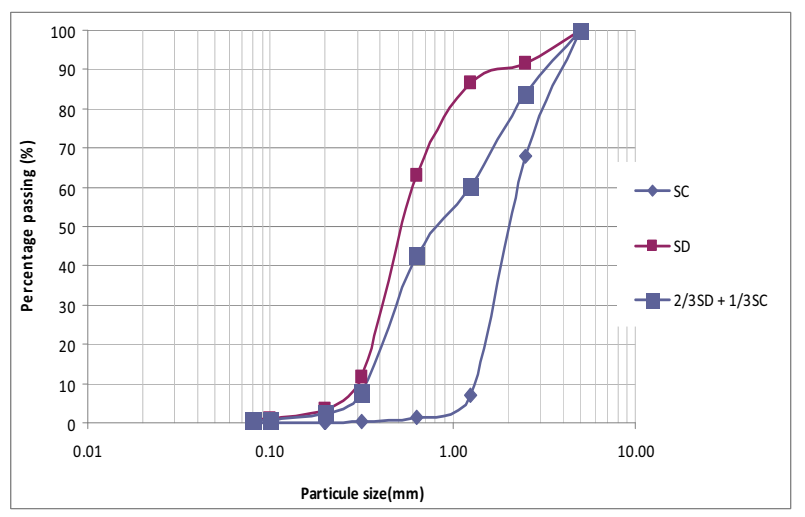

Fig. 1. L'analyse granulométriques des sables

\section{Formulation et composition de béton}

Nous avons réalisé 3 formulation de béton à haute performance en utilisant les trois nature de sable et en considérant deux rapports eau / ciment ( $\mathrm{E} / \mathrm{C}$ )égale à (0.3) . 
Les compositions des bétons sont obtenus suivant la méthode de la méthode de formulation des BHP de l'université de Sherbrooke, qui permet de formuler des BHP sans air entraîné conformément à la norme ACI 211-1 [Aitcin 2001]. Les affaissements au cône d'Abrams des bétons étudiés les positionnements en $6 \mathrm{~cm}$. Nous présentant dans le tableau 3 les compositions retenues des 3 bétons étudiés.

Tableau 3. Composition des bétons de l'étude (données pour $1 \mathrm{~m}^{3}$ )

\begin{tabular}{|c|c|c|c|}
\hline & Béton 1 & Béton 2 & Béton 3 \\
\hline $\begin{array}{c}\text { Sable de dune } \\
\text { SD }\end{array}$ & 701.48 & & 467.65 \\
\hline $\begin{array}{c}\text { Sable concassé } \\
\text { SC }\end{array}$ & $/ /$ & 701.48 & 233.83 \\
\hline Graviers 3/8 & \multicolumn{3}{|c|}{312.18} \\
\hline Graviers 8/15 & \multicolumn{3}{|c|}{728.42} \\
\hline Ciment C & \multicolumn{3}{|c|}{23.5} \\
\hline Fume de silice & \multicolumn{3}{|c|}{121.43} \\
\hline E & \multicolumn{3}{|c}{18.75} \\
\hline SP &
\end{tabular}

Les conditions de conservations des éprouvettes (10X10 $\mathrm{X} 10) \mathrm{cm}$ sont réalisées selon la recommandation de la RILEM TC-129. Toutes les éprouvettes sont conservées à une température de $20 \pm 5^{\circ} \mathrm{C}$ pendant les 24 heures après le coulage dans leurs moules sans échange hydrique avec l'extérieur. Les éprouvettes sont âgées de 28jours au moment des essais.

\section{Cycles de chauffage et refroidissement}

Les éprouvettes de béton subissent des cycles de chauffages refroidissement composés d'une phase de montée en température d'une phase de stabilisation à température constante et d'une troisième phase de descente de température jusqu'a température ambiante les rampe de chauffage et refroidissement utilisent la même vitesse de $10^{\circ} \mathrm{c} / \mathrm{min}$

\section{Résultats et discussions}

\subsection{Perte de masse du béton chauffé}

L'exposition des bétons aux hautes températures entraîne une dégradation des granulats et de la pâte de ciment dont l'une des conséquences est la diminution de la densité du béton. La perte de masse permet notamment de quantifier l'eau libre et liée présentes avant et après chauffage dans une éprouvette de béton. Cette eau se trouve sous forme liée dans les hydrates, absorbée à la surface des éléments solides et libre dans les pores. L'eau libre a la propriété d'être perdue facilement lors de variations thermiques [Baro 1994].
La valeur de perte de masse est une moyenne des mesures effectuées sur 3 éprouvettes de dimension $(10 \times 10 \times 10) \mathrm{cm}$ pour chaque type de béton .

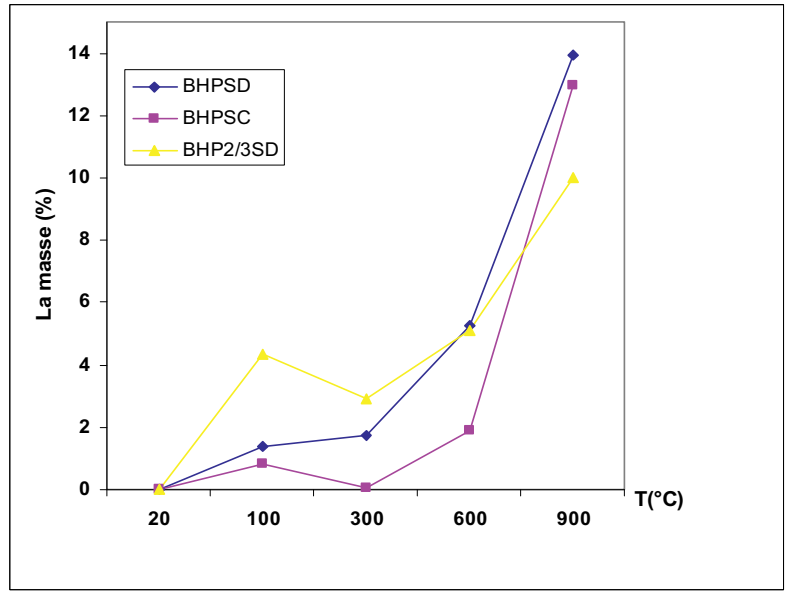

Fig. 2. Perte de masse des bétons testés en fonction de la température de chauffage.

BHPSD: Béton à haute performance formulé avec sable de dune (nature siliceux);

BHPSC: Béton à haute performance formulé avec sable de carrière (nature calcaire) ;

BHPSC: Béton à haute performance formulé avec $1 / 3$ sable de carrière plus $2 / 3$ sable de dune (nature calcaire siliceux);

Pour une matrice de béton à haute performance, le volume de pâte était constant pour les trois nature de granulats utilises dans les bétons (calcaire, silico-calcaire et siliceux). La différence de perte de masse après chauffage entre les bétons est donc seulement liée à la nature des granulats utilisés.

La perte de masse des différents bétons est très similaire jusqu'à $300^{\circ} \mathrm{C}$. Au-delà de cette température, la nature minéralogique des granulats influence la perte de masse des bétons (figure 2). Sur la totalité de l'eau qui part des bétons à hautes performances une proportion plus importante est contenue dans les granulats, compte tenu de faible rapport $\mathrm{E} / \mathrm{C}$ de la pâte. Ceci explique pourquoi dès $300^{\circ} \mathrm{C}$ des différences de pertes de masses apparaissent en fonction de la nature du granulats. Les différences de perte de masse entre les trois types de béton à hautes performances à $300^{\circ} \mathrm{C}$ peuvent être expliquées par l'eau libre dans les granulats. Les bétons BHP avec sable concassé présentent la plus faible perte de masse par rapport aux autres types de béton (jusqu'à $600^{\circ} \mathrm{C}$ ). La plus grande perte de masse des bétons de granulats silico- calcaire (jusqu'à $600^{\circ} \mathrm{C}$ ) peut s'explique en partie par la quantité d'eau absorbée par les carbonates poreux présents dans ce granulat et le départ d'eau liée de 
silex après $450^{\circ} \mathrm{C}$ [Zhi 2011]. Le départ de $\mathrm{CO}_{2}$ provenant des carbonates de calcium entraîne une nouvelle augmentation de la perte après $600^{\circ} \mathrm{C}$ pour les bétons gâchés avec le granulat calcaire BHPSC.

\subsection{Résistance résiduelle en compression}

La figure 3 présente les résistances résiduelles en compression obtenues à $20^{\circ} \mathrm{C}$ et après les différents traitements thermiques pour l'ensemble des bétons testés.

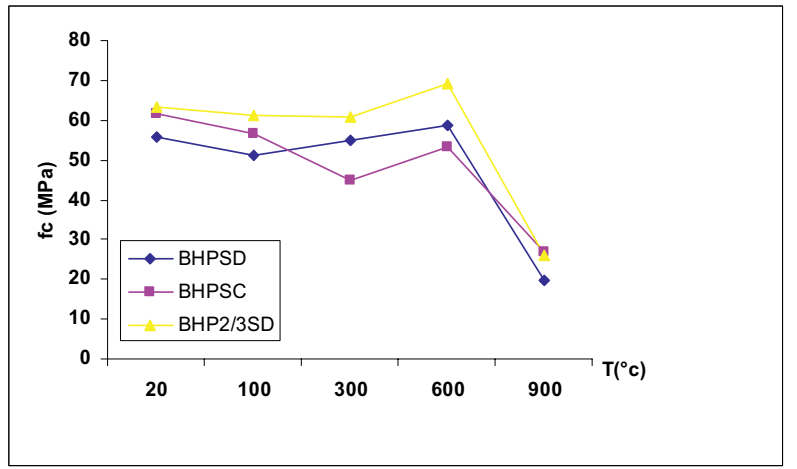

Fig. 3. Résistance résiduelle en compression des bétons à hautes performances en fonction de la température de chauffage

A température ambiante, la résistance ne varie pratiquement pas avec la nature des granulats. Elle est d'environ 70 et $80 \mathrm{MPa}$ pour les bétons à hautes performances. On observe sur la figure 3 deux zones d'évolution différentes selon la température de chauffage; un maintien de la résistance en compression jusqu'à $300^{\circ} \mathrm{C}$ puis une diminution continue avec l'augmentation de la température. Ceci rejoint les observations faites par d'autres chercheurs comme Dias [Dias 1990], Phan [Phan 2003] et Kanéma [Kané 2007].

Les bétons à hautes performances (figure 3), l'évolution de la résistance résiduelle en compression est similaire pour les trois bétons BHPSD, BHPSC, $\mathrm{BHP} 2 / 3 \mathrm{SD}$ jusqu'à $300^{\circ} \mathrm{C}$. A $600^{\circ} \mathrm{C}$ les bétons BHPSD BHPSC conservent plus de $50 \%$ de leur résistance initiale. Le béton de granulats calcaire présente toujours la plus grande perte de résistance en compression.

La différence entre la perte de la résistance de $\mathrm{BHPSD}$ et $\mathrm{BHP} 2 / 3 \mathrm{SD}$ est de $12 \%$ à $900^{\circ} \mathrm{C}$. La perte de résistance en compression des bétons de granulats siliceux à hautes performances reste modérée jusqu'a $600^{\circ} \mathrm{C}$ et s'accélère au-delà de cette température. Le meilleur comportement en compression de BHPSD peut être expliqué par une amélioration de la résistance de l'adhérence à l'interface pâte granulat. Le faible rapport eau / ciment de la pâte conduit en effet à une diminution de la porosité de la zone de transition. On peut noter que l'augmentation du volume total des granulats siliceux (quartzite) avec la transformation du quartz $\beta$ à $573^{\circ} \mathrm{C}$ ne conduit pas à une diminution de performances des bétons de granulat siliceux par rapport aux bétons de granulats calcaire. La perte de résistance en compression du béton à hautes performances avec des granulats silico-calcaire entre 600 et $900^{\circ} \mathrm{C}$ est due à l'écaillage et l'éclatement des silex.

\section{Conclusions}

Ce travail présente une analyse thermiques et mécanique, de béton de différents granulats exposés à des cycles de chauffage de $10^{\circ} \mathrm{C} / \mathrm{min}$. trois types de granulats sont testés : un granulat siliceux ; un granulat silico-calcaire et un granulat concassé calcaire.

L'analyse de l'état de fissuration après les différents cycles thermiques a montré que la plupart des fissures sont en relation avec les granulats. Elles sont tangentielles à l'interface pâte /granulats, radiales entre granulats proche et transgranulaires. $\mathrm{A} 300^{\circ} \mathrm{C}$, pour tous les bétons, les fissures sont peu visibles à l'œil nu. Elle sont isolées et situées essentiellement à l'interface pate granulat. $\mathrm{A} 600^{\circ} \mathrm{C}$, l'état de dégradation des bétons est très différent selon la nature du granulat.

Pour les trios types de granulats, les fissures dans les bétons à hautes performances ont de plus faibles ouvertures.

Pour un rapport E/C de 0.3 , les différences de perte de masse selon la nature des granulats apparaissent déjà à $300^{\circ} \mathrm{C}$.

\section{References bibliographiques}

1. [Aitcin 2001] Aitcin C P. 2001. 'Bétons Haute Performance', Edition, Eyrolles, Paris.

2. [Dias 1990], WPS Dias, GA Khoury, PJE Sullivan, Mechanical properties of hardened cement paste exposed to temperature up to $700^{\circ} \mathrm{C}$, ACI Material journal ,87(1990) 160-166.

3. [Kané 2007], M. Kanéma, Influence des paramètres de formulation sur le comportement à haute performance des bétons, thèse de doctorat, université de GergyPontoise (2007).

4. [Phan 2003] L. T. Phan, N. J. Carino, Code provisions for high strength concrete strength-temperature relationship at elevated temperatures, Materials and Structures, March 2003, Volume 36, Issue 2, pp 91-98

5. [Zhi 2011] Xing, Zhi., Beaucour, A. L., Hebert, R., Noumowe, A., \& Ledesert, B. (2011), Influence of the nature of aggregates on the behaviour of concrete subjected to elevated temperature. Cement and Concrete Research, 41(4), 392-402. . 